\title{
Detection of short fatigue cracks by inductive thermography
}

by B.Oswald-Tranta, C. Tuschl,

\author{
Chair of Automation, University of Leoben, Peter-Tunnerstr.27, 8700 Leoben, Austria, \\ beate.oswald@unileoben.ac.at
}

\begin{abstract}
Inductive thermography can be used to detect surface cracks in metals. As a crack influences the eddy current distribution and the heat diffusion, it becomes visible in the infrared images. The deeper the crack, the larger disturbance it causes, hence its signal correlates with its depth. This paper investigates, how by short cracks their length influences the signal. Simulation and experimental results are presented for ferro-magnetic and for austenitic steel. The signal along the crack, between the hot spots at the crack tips, corresponds with its depth. It is also investigated how the crack profile affects this pattern.
\end{abstract}

\section{Introduction}

In the case of inductive thermography the workpiece is heated with a short $(0.1-1 \mathrm{~s})$ inductive heating pulse and an infrared camera records during and after the heating the temperature distribution at the sample surface. The induced eddy current decays exponentially below the surface and the coefficient of this exponential decay is called the skin depth or penetration depth. For its value it is valid:

$$
\delta=\sqrt{\frac{1}{\pi \mu_{0} \mu_{r} \sigma f}}
$$

where $\mu_{0}$ denotes the permeability of vacuum with the value of $4 \pi 10^{-7} \mathrm{Vs} / \mathrm{Am} ; \mu_{r}$ is the relative magnetic permeability of the material; $\sigma$ is its electrical conductivity and $f$ is the excitation frequency. For austenitic steel the skin depth is about $1.35 \mathrm{~mm}$, if the excitation frequency is $200 \mathrm{kHz}$. On the other hand, for the ferro-magnetic steel mainly due to its magnetic property with $\mu_{r}=600$, for the same excitation frequency this depth is only $0.034 \mathrm{~mm}$.

A surface breaking crack disturbs the eddy current distribution as the current flows around it [1,2], causing a local inhomogeneous heating around the crack. Additionally, the heat flux equalizing the temperature difference, is also affected by the crack. Due to these two effects surface cracks can be excellently detected with this technique in metallic surfaces [1-7].

Inductive heating has the advantage that the eddy current and therefore the heating itself is generated directly in the material, and it is not affected by the absorption coefficient of the surface, which is a usual problem by optically excited thermographic inspection. But the emissivity value has an influence on the thermal radiation detected by the infrared camera. In order to reduce the effect of the inhomogeneous emissivity and the effect of the inhomogeneous heating, usually a phase value is calculated by Fourier transform from the whole infrared sequence of the heating and cool down phase [1]. The phase image has a significantly higher signal-to-noise ratio than the temperature contrast around a crack.

The deeper of a crack, the larger obstacle it creates for the eddy currents and for the heat flux, therefore the signal around a crack increases with its depth. This dependency on the crack depth has been intensively investigated in the last years for long cracks [1,2]. In this paper short cracks are examined, how their length at the surface influences the typical 'butterfly' pattern obtained by evaluating the infrared image sequence.

\section{Simulation for short cracks}

FEM simulations were carried out using the multiphysics simulator ANSYS [8], to model the eddy current distribution, the generated Joule heating and the temperature distribution around short vertical surface cracks. Fig. 1 compares results for austenitic and for ferro-magnetic steel by an excitation frequency of $200 \mathrm{kHz}$. The simulated crack is $l=10 \mathrm{~mm}$ long and $d=1 \mathrm{~mm}$ deep. As the eddy current penetration depth in ferro-magnetic steel is very small compared to the crack depth $(\delta=0.034 \mathrm{~mm})$, hence the eddy currents follow the line of the crack, see Fig. $1 \mathrm{a}$. The whole crack surface is selectively heated, causing along the line of the crack a higher temperature [1,2]. Additionally, around the crack tips a high current density occurs, hence here a hot spot can be observed in the temperature image, see Fig.1b.

In the austenitic steel for the same excitation frequency the eddy current penetration depth is larger than the crack depth $(\delta=1.35 \mathrm{~mm}, d=1 \mathrm{~mm})$. The current lines are deflected by the surface cracks (see Fig.1c), therefore the current density at the surface close to the crack line is reduced and the Joule heating and the temperature are less in this region than at the sound, faultless surface $[1,2,9]$, see Fig.1d. But around the crack tip at the surface the same phenomena can 
be observed as by the ferro-magnetic steel: the eddy current flows around the crack tips, causing in the vicinity of the crack tips higher current density, higher Joule heating and hot spots in the temperature distribution.
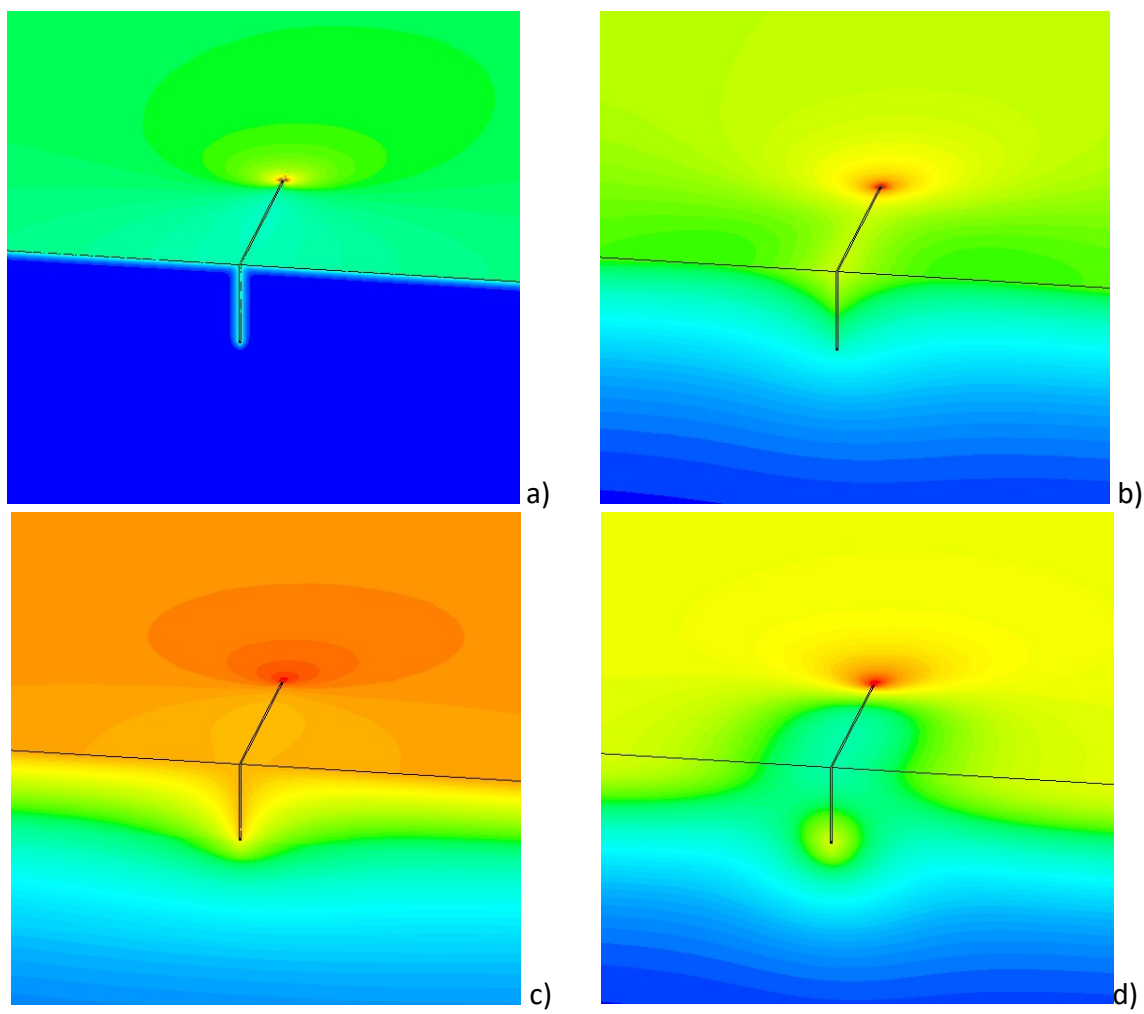

Fig. 1. Magnitude of the magnetic field (a, c) around a $10 \mathrm{~mm}$ long and $1 \mathrm{~mm}$ deep crack; and the temperature distribution after $0.1 \mathrm{~s}$ inductive heating pulse with $200 \mathrm{kHz}$ excitation frequency $(b, d)$. Figs. a and $b$ are calculated for ferro-magnetic steel; $c$ and $d$ for austenitic steel. The images show only the half of the model in order to see also the distribution below the surface in the middle of the crack.

Figs.2a and $2 \mathrm{~b}$ show for the same models the temperature distribution at the surface. The magnetic field lies in the $\mathrm{x}$-direction, and the eddy currents flow perpendicular to it into the $y$-direction. It is well to observe that in the case of ferro-magnetic steel in the mid-range of the crack the temperature is higher than at the sound surface far away from the crack. On the other hand, in the case of the austenitic steel the temperature is less in this region.

In the mid-range of the crack the eddy current flows below the crack. But in the vicinity of the crack tips the currents flow at the surface around the tip, causing in this region a higher eddy current and Joule heating density. In the corresponding phase images (Fig.2c and in Fig.2d) the crack tips have the highest phase values, because the temperature during the heating most rapidly increases in the vicinity of the crack tips and in the cool-down phase the temperature most rapidly decreases here. But in the mid part of the cracks the same phase distribution can be observed as for long cracks. This is only valid, if the crack is not too short. In the case of cracks, as shown in Figs.2e and $2 f$ for $2 \mathrm{~mm}$ long cracks, the hot spots at the crack tips affect the phase distribution also in the middle of the crack, and in both cases for ferro-magnetic and non-magnetic steel the phase value in the mid range is less than at the sound surface. 

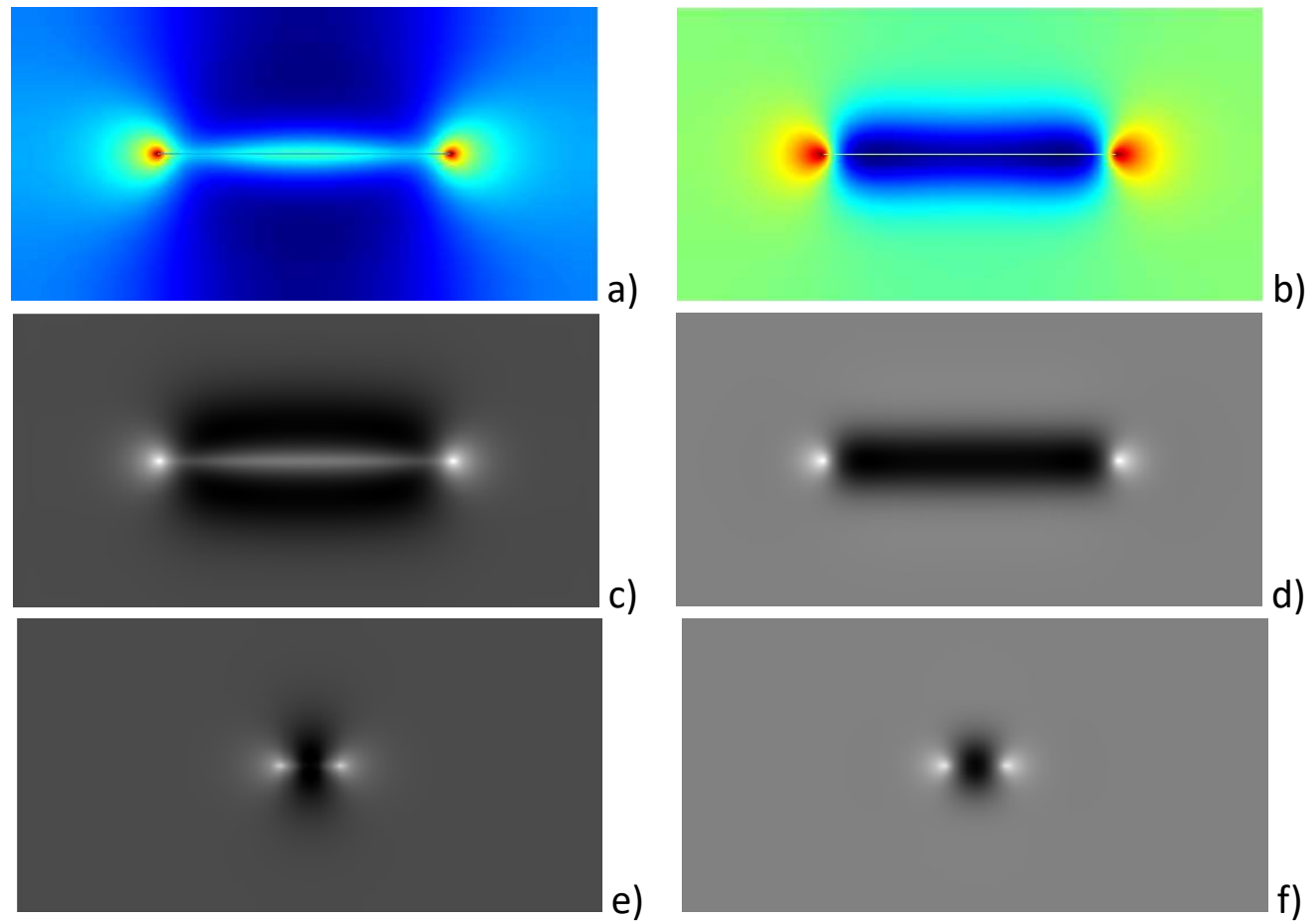

Fig. 2. Temperature distribution after $0.1 \mathrm{~s}$ heating around a $10 \mathrm{~mm}$ long and $1 \mathrm{~mm}$ deep crack in ferromagnetic steel (a) and in non-magnetic steel (b); phase images for the same cracks: fig.c in ferro-magnetic steel and fig.d in austenitic steel; phase images for a short crack with $2 \mathrm{~mm}$ length and $1 \mathrm{~mm}$ depth in ferro-magnetic steel (e) and in austenitic steel (f).

\section{Influence of crack length and crack depth}

In Fig. 3 simulation results for different crack lengths in the range of $1 \mathrm{~mm}$ up to $12 \mathrm{~mm}$ are depicted. In the phase profiles along the crack lines the hot spots at the crack ends are for both materials well visible. In these hot spots additional heat is generated, which flows to the surrounding region, causing there a slower temperature decrease. This heat accumulation becomes in the phase image visible due to a lower phase value. If the crack length is shorter than this affected range around the crack tip, then the phase value in the middle of the crack is also influenced by the heat flow from crack tips and the phase value becomes different from the value which could be observed for a long crack. From which crack length the phase value in the mid position of a short crack is the same as for a long crack, depends on different parameters:

- $\quad$ Heating pulse duration;

- $\quad$ Thermal diffusivity of the material;

- $\quad$ Crack depth.

But the range of this critical crack length is always in the range of $6-10 \mathrm{~mm}$ for both steel materials. In the case of a $1 \mathrm{~mm}$ deep crack in ferro-magnetic steel with $0.1 \mathrm{~s}$ heating pulse the crack length $8 \mathrm{~mm}$ shows the same behaviour in the middle of the crack as a long crack. For austenitic steel in the case of $1 \mathrm{~mm}$ deep crack and $0.1 \mathrm{~s}$ heating pulse duration the limit is about $6 \mathrm{~mm}$ length. These two curves are plotted with a thicker black line in Fig. 3. 

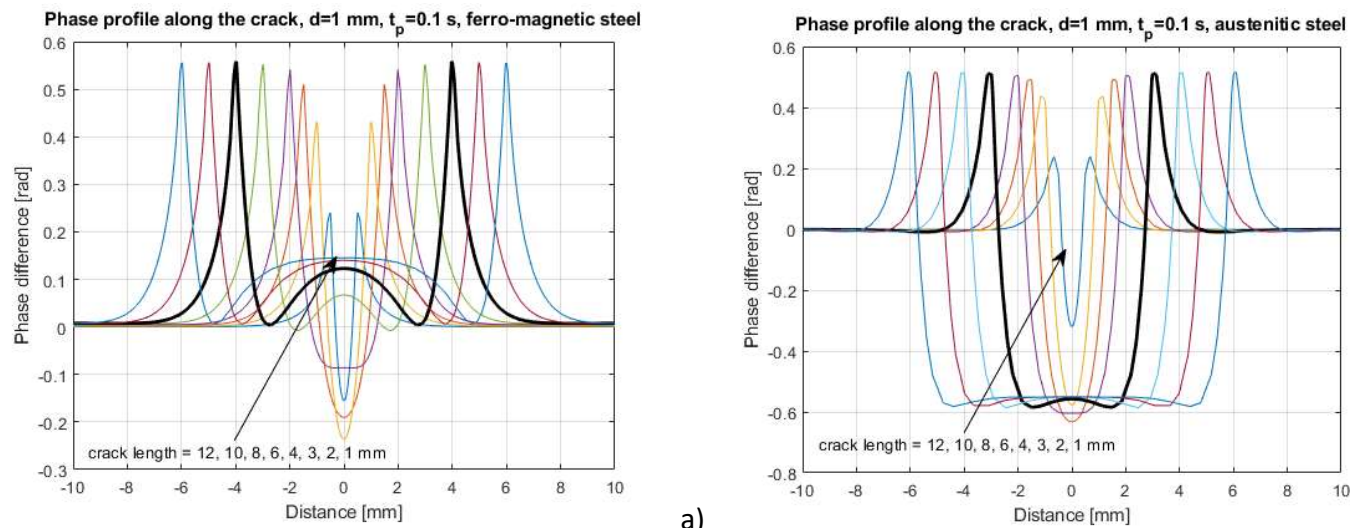

b)

Fig. 3. Phase profiles along the crack calculated for different crack lengths; heating pulse $=0.1 \mathrm{~s}$, crack depth $=1 \mathrm{~mm}$, in ferro-magnetic steel (a), austenitic steel (b). In both figures the line belonging to the shortest crack length where the phase value approximately equal to the phase value of a very long crack, is plotted by a thick black line.

The deeper is a crack, the larger obstacle it presents for the eddy currents and for the heat flow. Therefore, the phase contrast around a crack is increasing with the crack depth [1,2]. Fig. 4 compares the phase profiles across and along the cracks for different crack depths. Additionally, it can be noticed that not only the phase contrast in the mid position, but also the phase values at crack ends increase with the crack depth. In Figs.4c and 4d the phase profiles across the $10 \mathrm{~mm}$ long cracks are plotted together with the phases calculated for long cracks. The main difference in the modelling is, that long cracks can be simulated in a 2D model, in a cross-section across the crack. For short cracks the simulation model has to be setup in 3D, in order to consider the length of the crack. As in Figs.4c and $4 \mathrm{~d}$ can be seen, for the crack with a $10 \mathrm{~mm}$ length the phase in the mid position of the crack is almost identical with the long ones.

The phase contrast can be defined in two ways, as it is shown in Figs.5a and 5b:

$$
\begin{aligned}
& \Phi_{\text {contrast } 1}=\Phi_{\text {crack }}-\Phi_{\text {min }} \\
& \Phi_{\text {contrast } 2}=\Phi_{\text {crack }}-\Phi_{\text {sound }}
\end{aligned}
$$

Depending on which of these contrasts in absolute value is larger, it will be noticed in an image and used to characterize the crack:

$$
\text { if } \begin{aligned}
\left|\Phi_{\text {contrast } 1}\right|>\left|\Phi_{\text {contrast } 2 \mid}\right| \text {, then } \Phi_{\text {contrast }} & =\Phi_{\text {contrast } 1} \\
\text { else } \Phi_{\text {contrast }} & =\Phi_{\text {contrast } 2}
\end{aligned}
$$

According to this definition the phase contrast dependency on the crack length is compared in Fig.5.c:

- Very short cracks with length of 1-2 mm look for both materials and for the different crack depths similar: both hot spots at the end of the cracks are visible and between them the phase value is negative. This can be also seen in Figs.2e and $2 f$.

- Cracks with 2-6 mm length show also similar patterns with two hot spots and negative phase values between them. But this negative value in the middle of the crack is in absolute value larger than for significantly longer cracks, as the heat flow from the hot spots affects the phase value in such a short distance from the ends.

- Cracks larger than 6 or $8 \mathrm{~mm}$ has the same phase contrast as longer cracks, the length of the crack has no effect any more on the phase contrast. 
10.21611/qirt.2020.033

$15^{\text {th }}$ Quantitative InfraRed Thermography Conference, 6 - 10 July 2018, Porto, Portugal
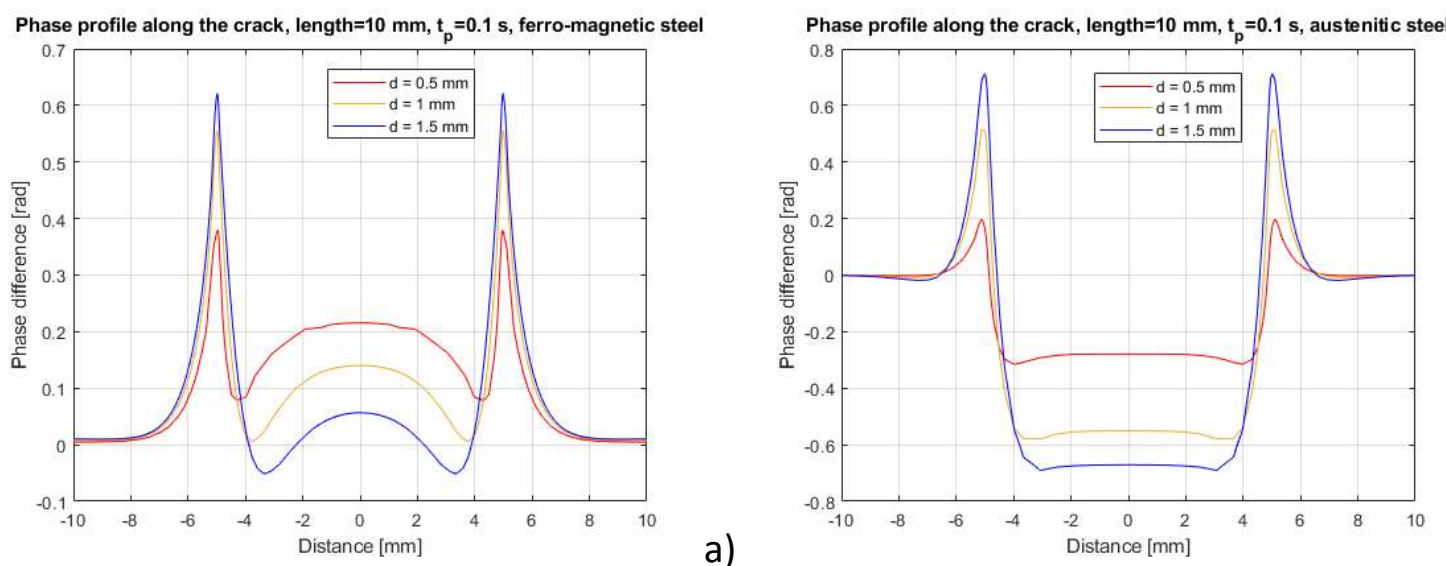

b)
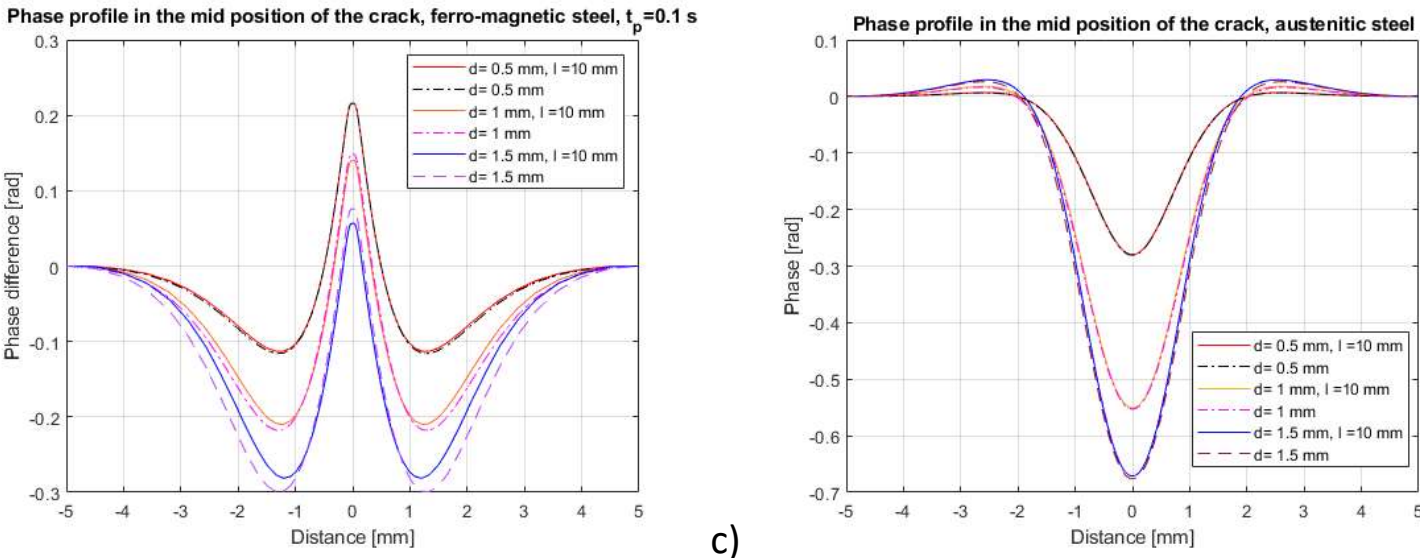

d)
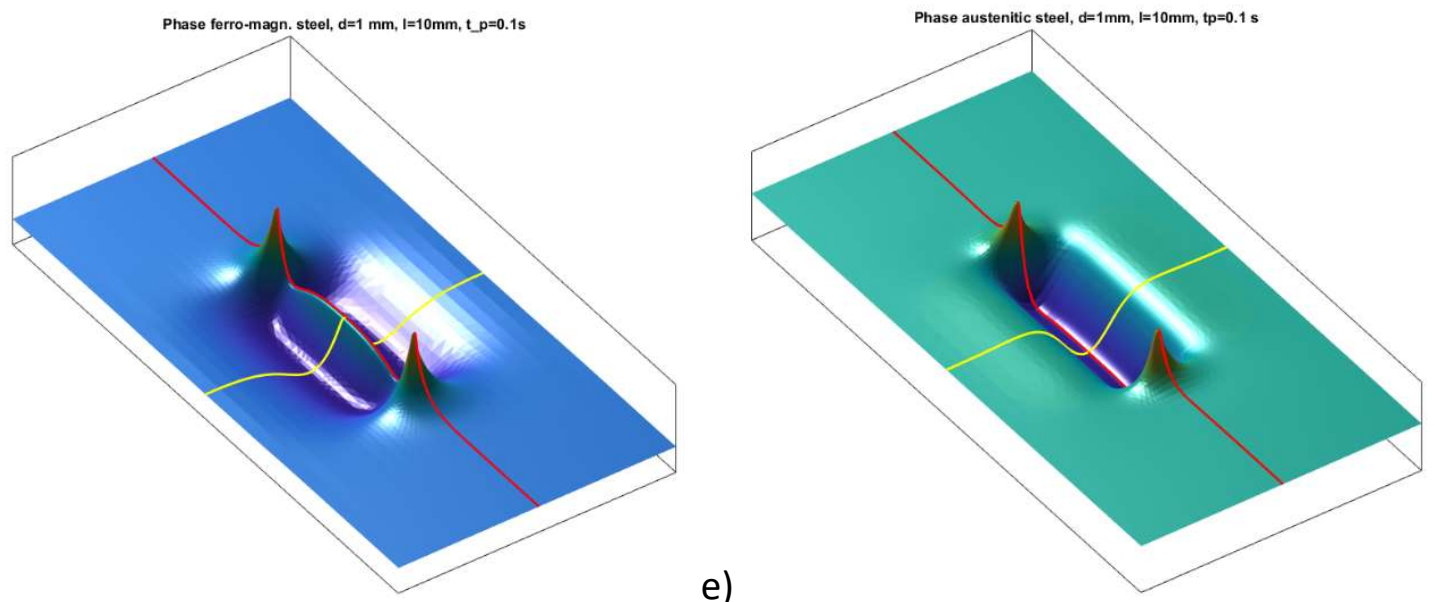

Fig. 4. Phase profiles along the crack calculated for different crack depths; heating pulse $=0.1 \mathrm{~s}$, crack length $=10 \mathrm{~mm}$, in ferro-magnetic steel (a), austenitic steel (b). In figures $c$ and d phase profiles across the cracks in the mid positions are compared with the ones across long cracks, these later ones are plotted by dashed lines; Figures e and f show the phase plotted as a surface with the definitions for the profiles: red lines mark the phase profile along the crack and yellow lines show for the phase profile across the crack in the mid position. 

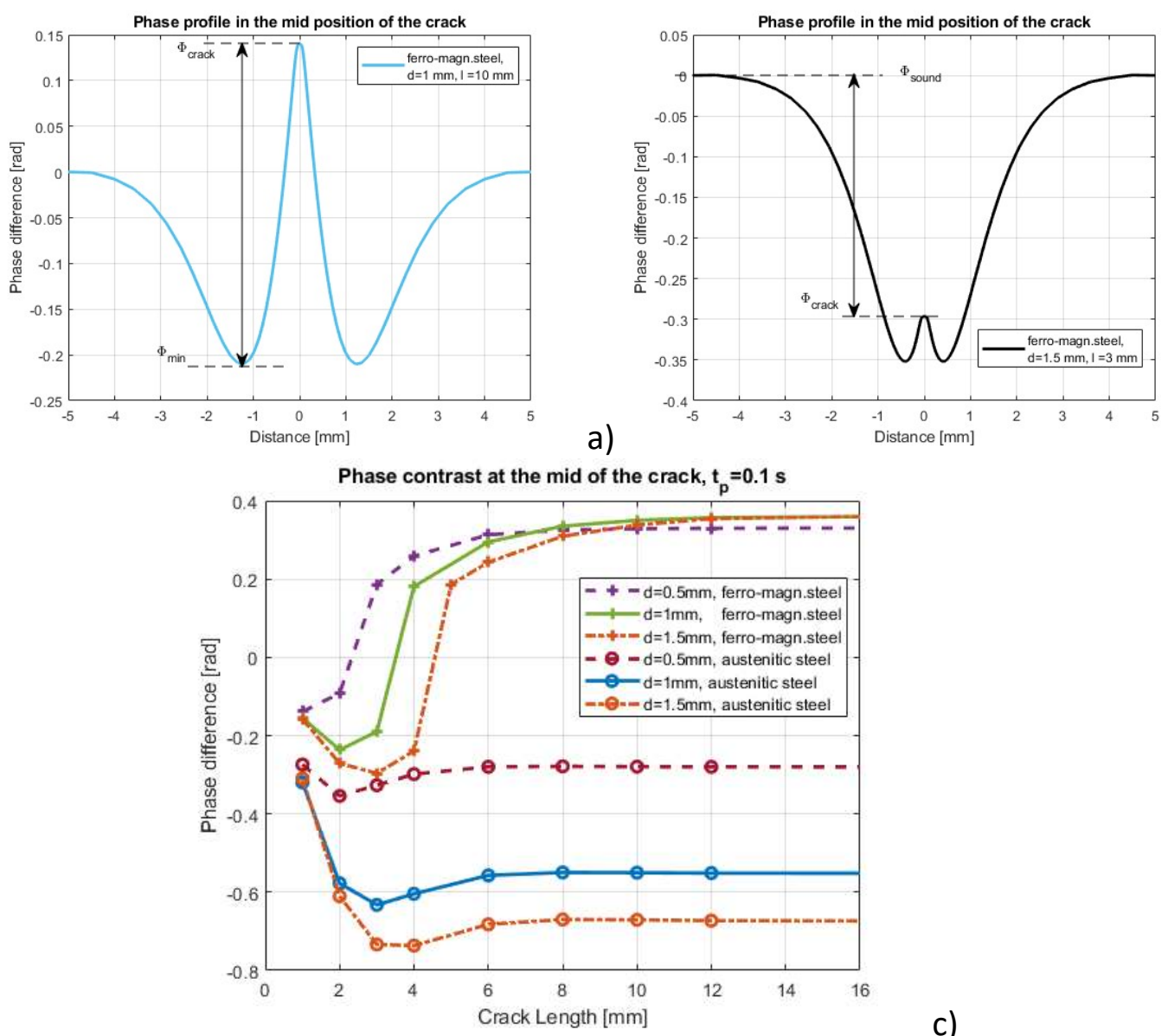

c)

Fig. 5. $a$ and b show two different ways to define the phase contrast; $c$ : phase contrast depending on the crack length, calculated for three different crack depths in ferro-magnetic steel and in austenitic steel.

\section{Influence of heating pulse duration}

Fig. 6 compares the phase profiles for different heating pulse durations. The eddy current distribution is disturbed by a crack causing an inhomogeneous Joule heating and an inhomogeneous temperature distribution. But the heat flow starts to equalize this inhomogeneity even during the heating pulse. The longer is the heating pulse, the more this temperature difference diminishes. This effect is stronger for the austenitic steel, as around the crack the temperature is lower and the heat flows to this position $[1,9]$. In the case of ferro-magnetic steel this effect is not so dominant as the crack is selectively heated and the heat is longer trapped by the crack edges.

It can be noticed in Fig. 6 how the heat diffuses from the hot spots away in the vicinity of the crack tips. By longer heating pulse the intensity of the peak is reducing and the size of the hot spot is increasing. 


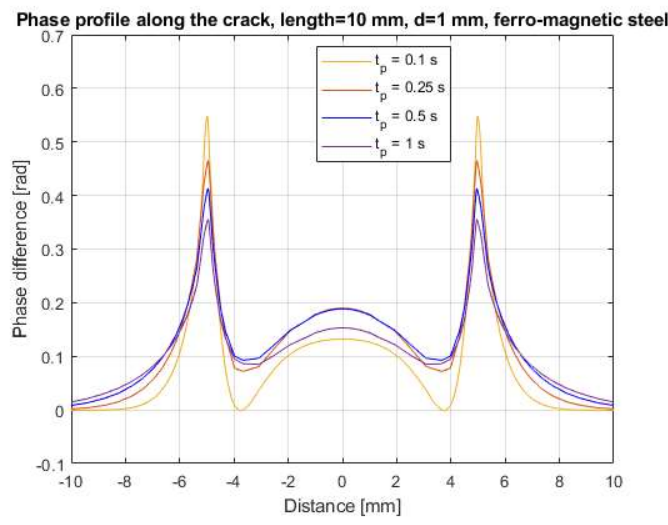

a)

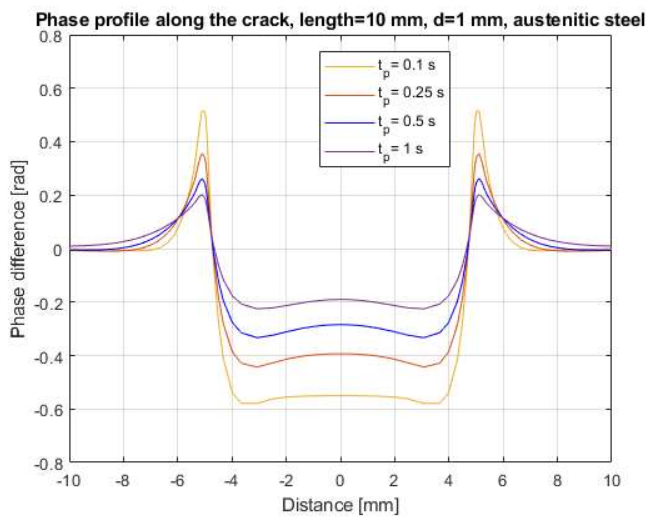

b)

Fig. 6. Phase profiles along the crack calculated for different heating pulse durations, crack depth $=1 \mathrm{~mm}$, crack length $=10 \mathrm{~mm}$, in ferro-magnetic steel (a) and in austenitic steel (b).

\section{Influence of crack profile}

All the simulated defects shown until now have a rectangular shape inside the material. Natural, fatigue cracks have usually a different profile, which is closer to a half-penny shape instead of a rectangle one [9]. In most of the cases the crack does not change abruptly to the maximum crack depth, but it is starting slowly until achieving the deepest point. In order to analyze the effect of the crack profile further simulations were calculated for cracks with trapezoid shape. Fig.7 and Fig. 8 show phase images, where the crack length at the surface is $10 \mathrm{~mm}$, but inside the material the length is shorter. The crack ends penetrate slanted into the material, having a trapezoid shape, see Fig.7c. Comparing the phase images and the phase profiles, it can be observed that the 'hot spots' at the crack tips are much less dominant for the trapezoid shaped cracks than for the rectangular ones. But the phase value in the mid part of the crack is approximately the same, as for the rectangular one, assumed that the crack is long enough.
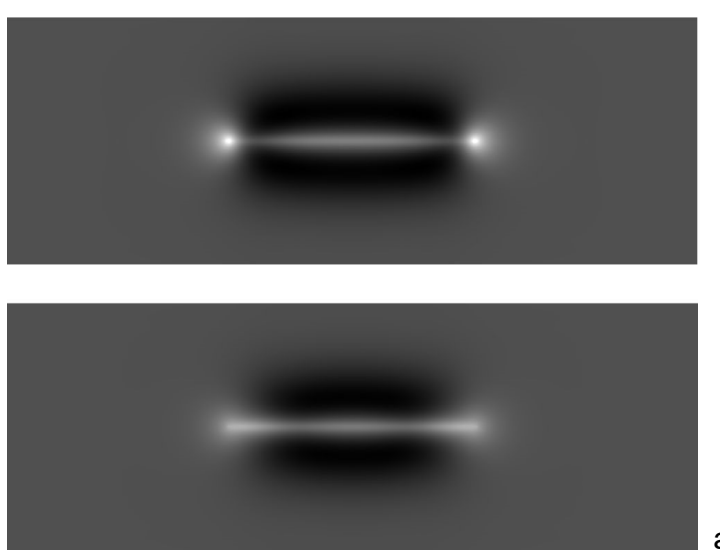

Phase profile along the crack, ferro-magn., $t_{p}=0.1 \mathrm{~s}, \mathrm{~d}=1 \mathrm{~mm}, \mathrm{l}=10 \mathrm{~mm}$

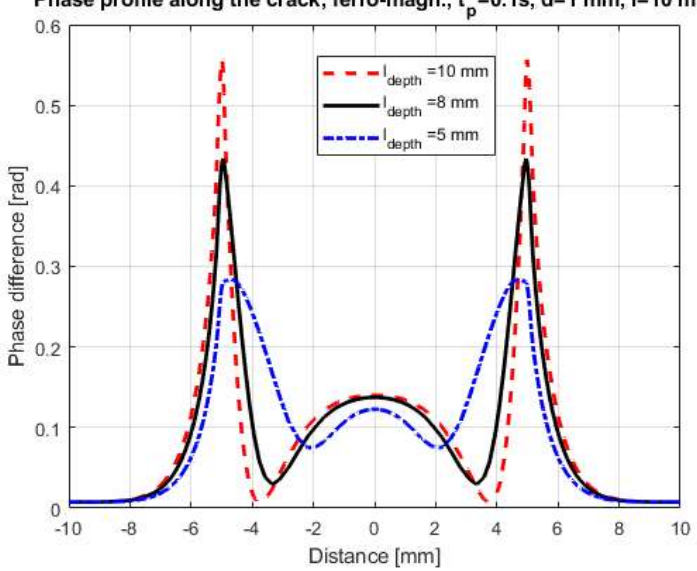

b)

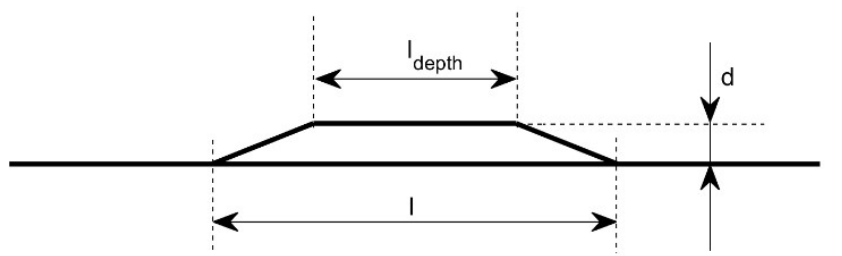

c)

Fig.7: Simulation results for $10 \mathrm{~mm}$ long and $1 \mathrm{~mm}$ deep vertical cracks in ferro-magnetic steel with different cross-section profiles; top a: rectangular profile, Idepth $=10 \mathrm{~mm}$, bottom a: Idepth $=5 \mathrm{~mm}$, fig. $b$ : comparing the profiles along the crack; fig. c: sketch of the trapezoid shaped crack with the length definitions. 

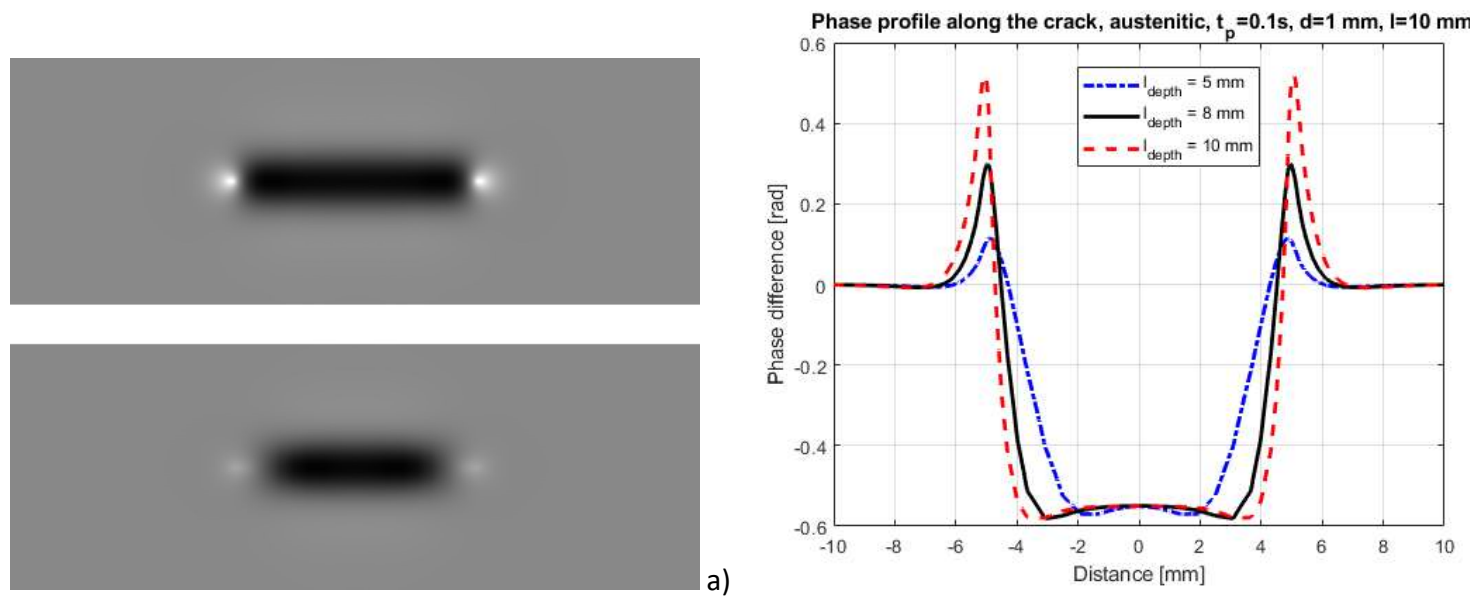

b)

Fig.8: Simulation results for $10 \mathrm{~mm}$ long and $1 \mathrm{~mm}$ deep vertical cracks in austenitic steel with different crosssection profiles; top a: rectangular profile, $I_{\text {depth }}=10 \mathrm{~mm}$, bottom a: $I_{\text {depth }}=5 \mathrm{~mm}$, fig. $b$ : comparing the profiles along the crack.

\section{Experimental results}

In the experiments a $10 \mathrm{~kW}$ induction generator was used and the excitation frequency was $200 \mathrm{kHz}$. The sample is placed in the middle of a Helmholtz coil [9] and the infrared camera records the surface temperature from the top. The infrared camera has a cooled InSb detector sensitive in the 1.5-5.1 $\mu \mathrm{m}$ mid-IR range. The recording frequency was $380 \mathrm{~Hz}$.

Two samples were used in the experiments to demonstrate the capability of the inductive thermography. In Fig.9a the sample is ferro-magnetic and the crack length is about $12 \mathrm{~mm}$ long and has approximately a depth of $1 \mathrm{~mm}$. In Fig.9b the sample is austenitic steel and the fatigue crack has approximately a half-penny shape and a depth of $1.5 \mathrm{~mm}$ [9]. Both cracks are natural cracks and not visible to naked eye due to their opening just in $\mu \mathrm{m}$ range. It was not necessary to blacken the surface to increase the emissivity, the measurements were carried out at the partially shiny surface. In both cases the cracks can be made well visible in the thermography measurement and due to the phase calculation the signalto-noise ratio in significantly higher, than in the temperature image. The hot spots at the end of the cracks can be well recognized, and measuring their distance the crack length could be determined. Comparing the phase value in the mid of the crack, the depth can be estimated as well. It is noticeable, that in the ferro-magnetic steel along the crack the phase value is higher than at the sound surface, similarly to the simulations in Fig.7a. On the other hand, in the austenitic steel, due to the larger eddy current penetration depth, the phase value along the crack is less than at the sound surface.
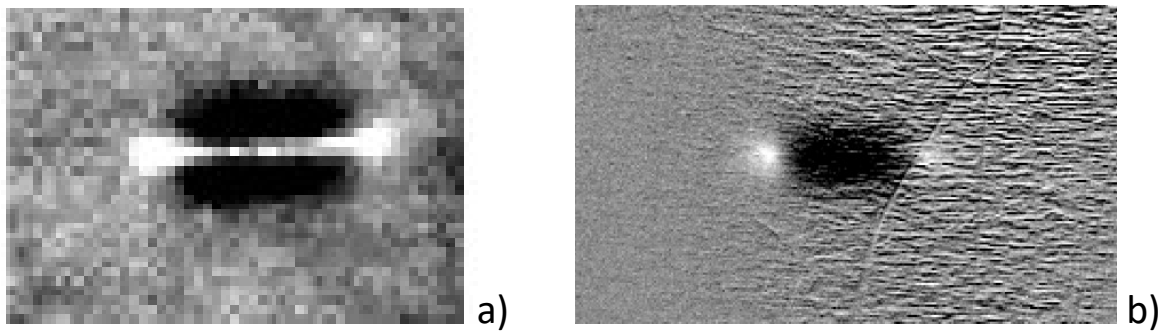

Fig. 9. Phase image for a $12 \mathrm{~mm}$ long crack in ferro-magnetic steel sample after $0.1 \mathrm{~s}$ heating pulse duration (a), and for a $6 \mathrm{~mm}$ long fatigue crack in austenitic steel after $0.25 \mathrm{~s}$ heating pulse duration (b) [9].

\section{Summary}

Short surface cracks in the range of 1-14 mm length were investigated in ferro-magnetic and also in austenitic, non-magnetic steel. Several simulations were carried out, where the eddy current distribution and the heat diffusion around the vertical cracks are calculated. The temperature sequence during and after the short heating pulse is evaluated by Fourier transform to a phase image. In earlier publications [1,2] the phase contrast was investigated for long cracks and how this contrast can be used for estimation of the crack depth. In the current paper the main goal was to study, whether this estimation can be also applied for short cracks and up to which length is this assumption valid.

The simulation results show that short cracks can be always well detected from the hot spots at the crack tips. The phase value between the hot spots for very short cracks (length appr. 1-2 mm) is in both materials lower than at the sound surface. If the crack is longer than about $8-10 \mathrm{~mm}$ than the phase value is similar to the long, with $2 \mathrm{D}$ simulation 
modelled or analytically calculated [1] phase contrast. Therefore these values can be also used for the depth estimation of the short cracks.

The phase value at the hot spots is not appropriate for depth estimation. The crack profile, whether it is rectangular, half-penny or trapezoid shaped, has a strong influence on the intensity of the hot spot, and this effect is more significant than the dependency on the crack depth.

In the current paper only two samples, one ferro-magnetic and one austenitic steel, were used to demonstrate the inductive thermography measurements for detecting short surface cracks. Further experiments are planned to be published in another publication.

\section{REFERENCES}

[1] Oswald-Tranta.B, "Induction Thermography for Surface Crack Detection and Depth Determination", Appl. Sci.8, 257; http://dx.doi.org/10.3390/app8020257 (2018)

[2] Oswald-Tranta, B. "Investigations for Determining Surface Crack Depth with Inductive Thermography," Proc. of the 19th World Conference on Non-Destructive Testing, Munich, Germany, 13-17 June 2016.

[3] Netzelmann, U.; Walle, G. Induction Thermography as a Tool for Reliable Detection of Surface Defects in Forged Components. In Proceedings of the 17th World Conference on Non Destructive Testing, Shanghai, China, 25-28 October 2008.

[4] Srajbr, C. Induction Excited Thermography in Industrial Applications. In Proceedings of the 19th World Conference on Non-Destructive Testing, Munich, Germany, 13-17 June 2016.

[5] Netzelmann, U.; Walle, G.; Lugin, S.; Ehlen, A.; Bessert, S.; Valeske, B. Induction thermography: principle, applications and first steps towards standardization. Quant. InfraRed Thermography J., 2016, 13, 170-181

[6] J.Vrana, M.Goldammer, "Defect detection mechanisms with induction and conduction thermography: current flow and defect-specific warming", Quantitative InfraRed Thermography,Journal (2019).

[7] DIN 54183:2018-02, Non-destructive testing-Thermographic testing- Eddy-current excited thermography; Available online: https://www.din.de (accessed on Jan.2018)

[8] ANSYS, Inc. Available online: http://www.ansys.com (accessed on 1 January 2018).

[9] Oswald-Tranta.B et al., „Comparison of different inspection techniques for fatigue cracks”, Proc. SPIE 11409, Thermosense: Thermal Infrared Applications XLII, 2020. 